\title{
A integração da tecnologia na educação básica e secundária em Portugal desde os anos 70 do século $\mathrm{XX}$ à contemporaneidade
}

The integration of technology in basic and secondary education in Portugal from the 70s of the 20th century to the present day

Integración tecnológica en la educación secundaria en Portugal desde la década de 1970 hasta la actualidad
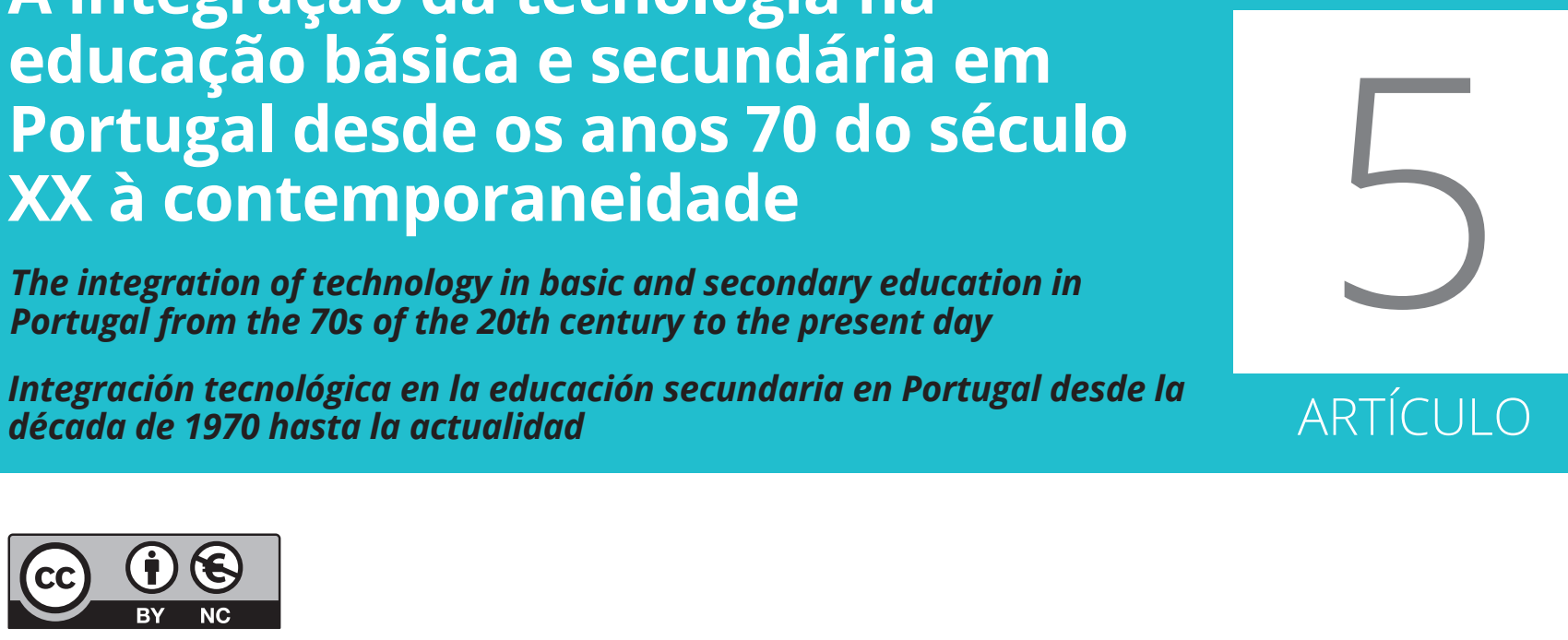

\section{Sara Dias-Trindade}

Universidade de Coimbra (Portugal)

Universidade de Coimbra, Centro de Estudos Interdisciplinares, Faculdade de Letras (DHEEAA). Doutora em História.

sara.trindade@uc.pt

orcid.org/0000-0002-5927-3957

\section{José António Moreira}

Universidade Aberta (Portugal)

Universidade Aberta, Departamento de Educação e Ensino a Distância, Centro de Estudos Interdisciplinares. Doutor em Ciências da Educação.

jmoreira@uab.pt

orcid.org/0000-0003-0147-0592

\section{António Gomes Ferreira}

Universidade de Coimbra (Portugal)

Universidade de Coimbra, Centro de Estudos Interdisciplinares, Faculdade de Psicologia e ciências da Educação. Doutor em Ciências da Educação.

antonio@fpce.uc.pt

orcid.org/0000-0002-3281-6819

RECEBIDO: 26 de abril de 2021 / ACEITO: 28 de julho de 2021

OBRA DIGITAL, Núm. 21, Septiembre 2021 - Enero 2022, pp. 93-112, e-ISSN 2014-5039 DOI: https://doi.org/10.25029/od.2021.319.21 


\section{Resumo}

Este trabalho de revisão sistemática da literatura visa analisar as experiências pedagógicas com recurso a tecnologias em Portugal, entre os anos setenta do século XX e a atualidade, procurando compreender como foram sendo introduzidas na escola ao longo de cerca de cinquenta anos. Foram selecionados e analisados 47 artigos das bases de indexação SCOPUS, SCIELO e Web of Science. Verificou-se que, apesar de algum conservadorismo ainda na utilização da tecnologia, muito centrada no seu uso instrumental, nos anos mais recentes têm-se concretizado mais práticas com intencionalidade pedagógica e mais focadas na participação ativa dos estudantes. Constatou-se também a importância crescente da formação docente neste domínio das tecnologias digitais, com o objetivo de tornar os professores mais competentes e fluentes no uso pedagógico do digital.

\section{PALAVRAS-CHAVE}

Portugal, Tecnologia, Digital, Educação, Ensino básico e secundário.

\section{Abstract}

This systematic literature review aims to analyze the pedagogical experiences with the use of technologies in Portugal between the seventies of the twentieth century and today. It sought to understand how they were introduced into school over fifty years. A total of 47 articles were selected and analyzed from the SCOPUS, SCIELO and Web of Science indexing databases. It was found that, despite a certain conservatism very focused on the instrumental use of technology, more practices with peda- gogical intent and more focused on the active participation of students have been implemented in recent years, highlighting the growing importance of teacher training in this field of digital technologies, with the aim of making teachers more competent and fluent in the pedagogical use of digital tools.

\section{KEYWORDS}

Portugal, Technology, Digital, Education, Basic and secondary education.

\section{Resumen}

Este trabajo de revisión sistemática de la literatura, tiene como objetivo analizar las experiencias pedagógicas con el uso de tecnologías en Portugal, entre los años setenta del siglo XX y la actualidad, tratando de comprender cómo se introdujeron en la escuela a lo largo de unos cincuenta años. Se seleccionaron y analizaron 47 artículos de las bases de datos de indexación SCOPUS, SCIELO y Web of Science, y se encontró que, a pesar de cierto conservadurismo en el uso de la tecnología, muy centrado en su uso instrumental, en los últimos años se ha convertido en prácticas más materiales con vocación pedagógica y más enfocadas a la participación activa de los estudiantes, destacando la importancia creciente de la formación docente en este campo de las tecnologías digitales, con el objetivo de hacer que los docentes sean más competentes y fluidos en el uso pedagógico de lo digital.

\section{PALABRAS CLAVE}

Portugal, Tecnología, Digital, Educación, Educación básica y secundaria. 


\section{INTRODUÇÃO}

Numa relação socialmente complexa e não linear, a tecnologia tem influenciado a instituição escolar, que desde muito cedo foi acolhendo e transformando a sua própria cultura a partir daquilo que eram as novas possibilidades proporcionadas por diferentes artefactos tecnológicos que vieram contribuir, sobretudo, para melhorar o processo comunicativo. E, se por um lado, perante o crescimento do número de alunos, a escola precisava criar condições para se adaptar, por outro, o aumento da instrução acaba por estar intrinsecamente ligado ao desenvolvimento tecnológico.

Porém, ao se analisar, em particular o caso português, e como se foi processando, no início do século XX, a introdução de novidades tecnológicas na educação, verifica-se que estas novidades (o cinema, a rádio, variados equipamentos de áudio e, já na segunda metade do século, a televisão) acabaram por ter uma lenta difusão, de utilização esporádica, lúdica e quase exótica, e raramente integrada em práticas pedagógicas com o intuito de melhorar os processos de ensino e aprendizagem.

Esta situação resultava, sobretudo, da escassez de recursos da maior parte das instituições escolares, mas também era fruto de uma quase inexistente preocupação com formação docente para o uso pedagógico da tecnologia.

Assim, este trabalho tem como principal objetivo fornecer uma visão coetânea do uso e, sobretudo, da forma como a escola se apropriou da tecnologia a partir da introdução do ensino computorizado em Portugal. Nesse sentido, pretende-se analisar as experiências pedagógicas com recurso a tecnologias em Portugal, entre os anos setenta do século XX e a atualidade. Procurar-se-á compreender como foram introduzidas no contexto educativo português, que tipo de experiências pedagógicas foram sendo realizadas e, ainda, que preocupações têm existido no sentido de preparar os professores para o uso pedagógico dessas mesmas tecnologias. Será também analisado como ou de que forma se terão tornado "tecnologias educativas", no sentido atribuído por Silva (1989), ao destacar que "falar em tecnologia educativa significa, essencialmente, tornar o processo educativo mais eficaz e falar em eficácia significa melhorar a aprendizagem" (p. 39), ou Sarramona (1986) ao sublinhar a ideia de que se educar significava "fazer o homem", a tecnologia dava o seu contributo para o fazer cada vez melhor.

\section{A EVOLUÇÃO DA TECNOLOGIA NA ESCOLA: DAS “MÁQUINAS DE SOLETRAR" Às TECNOLOGIAS DIGITAIS}

É nos anos 60 do século XX que surgem os primeiros computadores e a primeira geração de "Computer Assisted Instruction" (CAl), que vão buscar a sua inspiração à máquina de Pressey. Porém, eram demasiados caros e não conseguiam atingir os objetivos esperados, nomeadamente, não conseguindo comprovar a sua utilidade em contexto educativo.

Na verdade, nos primeiros tempos a diferença entre os CAl e as antecessoras máquinas de ensinar estava no tipo de tecnologia para apresentar os materiais. Saettler (2004) explica que o estudante respondia às questões (preenchendo espaços ou assinalando a resposta certa), obtendo de imediato uma resposta. No caso de errar o sistema gerava de imediato nova questão e se a resposta estivesse correta, era apresentado mais material. Ou seja, uma atualização tecnológica da máquina de Pressey. 
Apesar de terem um valor muito elevado, a introdução destes primeiros computadores veio abrir a porta para novas experiências (Russel, 2006). Por isso, quando nos anos 70 do século XX o seu valor baixou o suficiente para tornar viável a sua introdução em ambiente escolar, houve até um certo forçar da sua introdução nas escolas norte-americanas por receio que os Estados Unidos ficassem para trás no que diz respeito aos avanços tecnológicos (Russel, 2006). Ensinar os estudantes a usar computadores parecia ser a solução para essa questão, bem como para assegurar que estes ficavam devidamente preparados para as novas exigências de uma sociedade cada vez mais tecnológica (Russel, 2006). Por último, o que seria o mais importante: os computadores poderiam contribuir para tornar o processo educativo mais eficiente.

Na realidade o que se foi verificando é que, pouco a pouco, entre os anos 60 e 80 do século XX, cada vez mais escolas começam a ter computadores, mas com um uso educativo muito precário.

Em 1960, começou a ser desenvolvido na Universidade de Illinois o PLATO (Programmed Logic for Automatic Teaching Operations), que permitia uma aprendizagem individualizada, a partir dos conteúdos previamente preparados pelo professor (Unwin \& McAleese, 1978).

Foi o primeiro computador com efetivo sucesso, seguido depois pelos produzidos pela IBM e pela Apple. A partir das ideias de Pressey e, sobretudo, de Skinner (1958), a IBM desenvolveu o computador IBM650, em 1953, sen-

1 É importante destacar que esta relação entre competências tecnológicas e competências para o trabalho é ainda hoje uma preocupação. Num estudo de 2015 do

Fórum Económico Mundial o gap tecnológico é apresentado como um dos motivos para maiores dificuldades em alguns países. do que a sua primeira experiência, enquanto equipamento de apoio ao ensino, foi realizada em 1959, para treino dos pilotos militares norte-americanos (Thammishetty, 2015). Não tardou a sua chegada às escolas.

O primeiro computador da IBM, produzido para uma escola, foi instalado em 1966 na Brentwood Elementary School em East Palo Alto. Em 1976 a Apple lança também o seu primeiro computador e, em 1978, ganha um concurso promovido pelo Minnesota Education Computing Consortium para equipar, com 500 computadores, as escolas do Estado.

No período do pós-primeira Guerra Mundial, as tecnologias começam a ser usadas enquanto "ajudas para o ensino", passando, já na década de 60 do século XX, a ser apelidadas de "ajudas para a aprendizagem", com a introdução do ensino programado que veio modificar o processo de ensino e aprendizagem em geral e a relação professor/aluno e otimizar os processos em sala de aula (Silva, 1993).

Silva (1993) indica que com as pesquisas educacionais desenvolvidas na década de 70, em conjunto com o desenvolvimento da cibernética e, nos anos 80 do século XX, dos hipermédia, ficava aberto o caminho para se poder falar em "tecnologia educativa".

Em Portugal, este período da focagem sistémica não se distancia cronologicamente do seu desenvolvimento a nível internacional, com um momento de arranque nos anos de 1960 e de afirmação já em plena década de 1970 e, sobretudo, durante os anos de 1980, caracterizado pela introdução da componente da tecnologia educativa na formação de professores, quer inicial, quer contínua, e acompanhada de projetos que se focavam precisamente na afirmação da tecnologia educativa como área estratégica do próprio sistema educativo (Silva, 1993). 
A preocupação com a formação de professores para o uso das tecnologias educativas foi crescendo sobretudo a partir de finais da década de 80 do século XX, acompanhando a reforma educativa portuguesa, e passando a estar integrada na componente da formação de professores, enquanto unidade curricular de diferentes cursos de licenciatura e de formação pós-graduada. Silva (2001) refere que "a Proposta Global da Reforma apresentada em 1989 incluía, no âmbito do plano da reorganização curricular e pedagógica três programas de execução que valorizam especialmente a implementação da Tecnologia Educativa no sistema educativo" (p. 245).

O primeiro programa passava pelo desenvolvimento de um primeiro projeto tecnológico no campo da educação, em 1985, intitulado MINERVA (Meios Informáticos no Ensino: racionalização/ valorização/ atualização), com o objetivo de aproximar as tecnologias do processo educativo. Já no início dos anos 90 iriam surgir os programas estatais, co-financiados pela Comunidade Europeia - Programa de Desenvolvimento Educativo para Portugal (PRODEP), que apetrecham as escolas de diversos recursos tecnológicos como retroprojetores, gravadores, fotocopiadores, televisores e, naturalmente, os primeiros computadores.

De então até hoje, têm sido variados os programas de digitalização da educação, sempre acompanhando os diferentes projetos que a União Europeia veio desenvolvendo, culminando no atual Plano de Ação para a Transição Digital, com uma área dedicada à educação, que se encontra em vigor desde 2020.

Se, até meados dos anos 80 do século XX, a introdução das tecnologias na educação era feita sem uma verdadeira preocupação pela sua incorporação em práticas pedagógicas e utilização enquanto recurso para potenciar os pro- cessos educativos, a partir de meio da década de 1980, e como refere Figueiredo:

a utilização educacional dos computadores começa agora a entrar numa fase de maturidade claramente apoiada em razões de natureza pedagógica, tanto a nível da concepção de suportes lógicos educacionais como ao nível das estratégias de utilização dos computadores nos ambientes formais e informais da aprendizagem escolar (1989, p. 80).

Os anos 90 do século XX viram chegar a Internet a Portugal com diferentes programas que foram disseminando os computadores nas diferentes escolas portuguesas. Nos anos 2000, os ambientes educativos reconfiguram-se, devido ao crescimento dos ambientes virtuais que vêm permitir novas interações, dentro da escola, com outras escolas, criando-se redes colaborativas e mesmo de "desterritorialização" da escola e dos processos educacionais.

Apoio à aquisição de diferentes equipamentos, quer por docentes, quer por discentes, apetrechamento das escolas com computadores, ligações à Internet, projetos, quadros interativos, formação docente e, também, desenvolvimento de programas de promoção da competência digital dos estudantes foram-se sucedendo (Figura 1). 


\section{Figura 1}

Timeline dos programas portugueses e europeus de tecnologias digitais em Educação

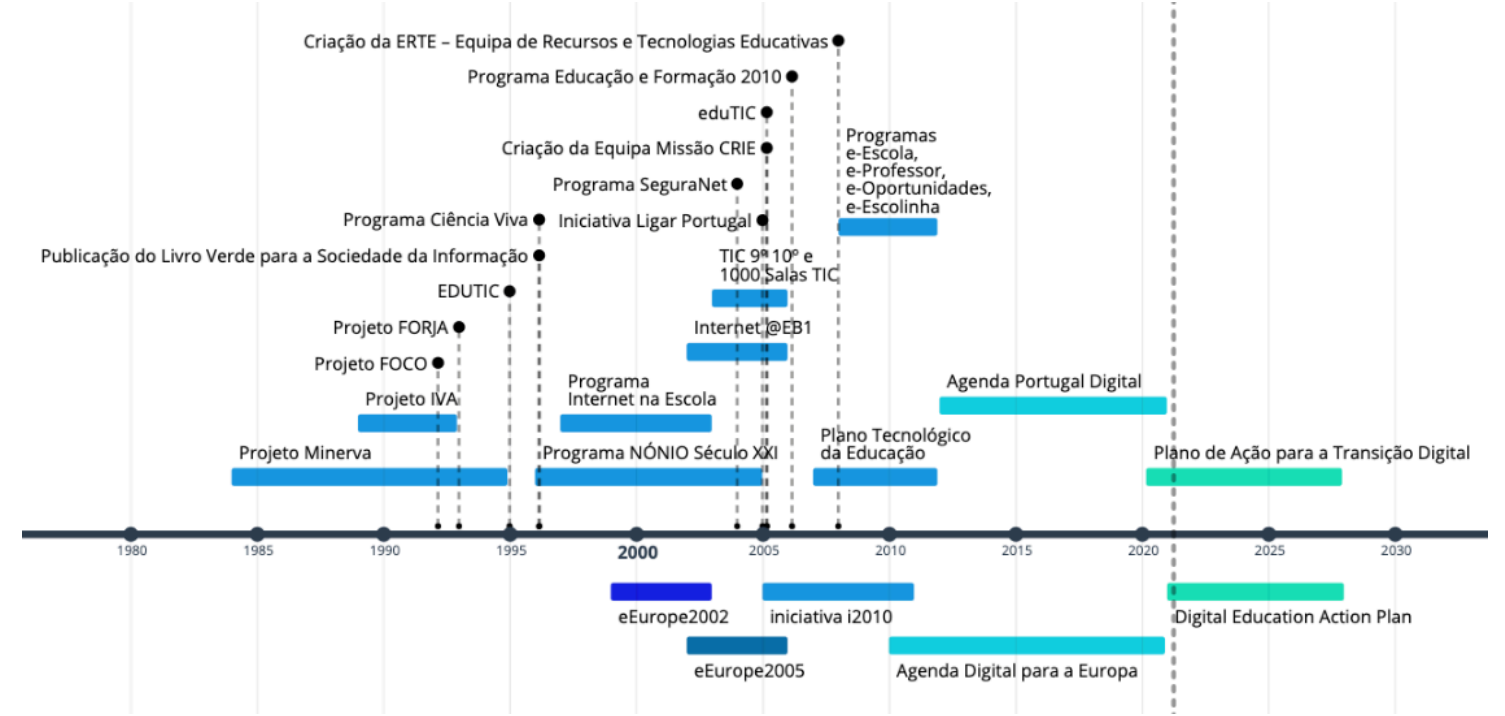

A rápida evolução dos equipamentos, sobretudo nos últimos 20 anos, e as possibilidades por eles trazidas, em particular a partir do desenvolvimento da Internet, modificou as práticas. De facto, "o foco da aprendizagem e das soluções metodológicas de ensino começam a integrar outros paradigmas pedagógicos, para além dos de base comportamental e cognitiva, mercê da circularidade permitida, em tempo real, à comunicação entre atores" (Rosenberg, 2001, pp. 25-29). Porém, Rosenberg refere que "a história da utilização de tecnologia eletrónica para a aprendizagem em meio escolar está repleta de promessas e desaires" (2001, p. 26), interrogando-se sobre se a Internet poderia modificar essa situação.

Por um lado, as tecnologias da informação e da comunicação passam, a partir das reformas curriculares portuguesas do início dos anos 2000, a integrar as práticas pedagógicas e a contribuir para que a cultura escolar tenha o seu papel na informatização da sociedade (Pinheiro \& Correia, 2014). Por outro, chega-se ao ano de 2020, com a obrigatoriedade de um ensino completamente online, durante os períodos de confinamento a que a pandemia de COVID19 votou as escolas, quer portuguesas quer internacionais, sendo visíveis as ainda existentes fragilidades, no processo de ensino e aprendizagem, em relação ao uso pedagógico das tecnologias digitais e dos recursos existentes (Dias-Trindade et al., 2020).

Apesar de tudo, considera-se que a disseminação da Internet veio permitir uma nova fase relativa à incorporação da tecnologia na escola, sobretudo a partir do desenvolvimento da web 2.0 e do desenvolvimento de um conjunto de recursos que veio permitir não só o desenvolvimento do trabalho colaborativo e em rede (Bassani \& Barbosa, 2018; Selwyn, 2010), referido anteriormente. Para além disso, contribuiu também uma mudança na cultura escolar, para uma era de "prod'utilizadores", ou seja, uma cultura em que os alunos participam de forma ativa, criam conteúdos que depois também utilizam, constroem e co-constroem conhecimento e ampliam os seus conhecimentos a partir das informações que vão recolhendo nas suas atividades educativas. 
Já no âmbito da pandemia COVID-19, o ConseIho de Ministros de Portugal aprovou o Plano de Ação para a Transição Digital, que se assume ser "o motor de transformação do país" (Conselho de Ministros, 2020, p. 8), e que visa pôr em prática um conjunto variado de medidas para proporcionar a sua digitalização. Estas medidas organizam-se em três pilares principais, sendo a primeira dedicada à formação e inclusão digitais, incluindo a educação digital e a requalificação e formação profissional.

No âmbito do ensino digital, um dos objetivos para a formação de jovens é a "integração transversal das tecnologias nas diferentes áreas curriculares do ensino básico e secundário" (Conselho de Ministros, 2020, p. 12). Esses objetivos procuram o desenvolvimento de uma formação transversal, adequada às competências para o século XXI, onde o papel da tecnologia, como já referido em 2015 pelo Fórum Económico Mundial (World Economic Forum [WEF], 2015), será fundamental para garantir a equidade social e profissional de todos no mundo. O sub-pilar que se refere à requalificação e à formação profissional especifica, entre as medidas a adotar, a formação de professores, o que, naturalmente, será essencial para que haja uma integração eficaz das tecnologias nas práticas educativas.

Com efeito, a qualificação dos professores é essencial para uma integração eficaz das tecnologias digitais nas escolas (Alves et al., 2019; Aşık, et al., 2020; Gutiérrez-Fallar \& Henriques, 2020; Ricoy \& Couto, 2011; Rodrigues, 2020), ao longo do processo educativo, de forma a criar uma cultura integrada e consciente do potencial que o digital tem no presente e no futuro dos jovens estudantes. Assim, o governo português está empenhado em acompanhar a realidade europeia, consciente de que as competências digitais são fundamentais para aumentar o grau de competitividade do país
(Conselho de Ministros, 2020; Governo Português, 2017; Ministério da Economia e da Transição Digital, 2020).

Porém, é importante compreender como é que em Portugal se veio construindo este percurso, como é que a entrada dos computadores nas escolas e, mais recentemente, a disseminação do sinal digital tem vindo a contribuir para a mudança de práticas.

\section{O ESTUDO}

O trabalho que aqui se apresenta assenta na metodologia de revisão sistemática da literatura, tendo-se seguido os procedimentos metodológicos recomendados por Petticrew e Roberts (2006).

\subsection{DELIMITAÇÃO DAS QUESTÕ̃ES DE INNVSTIGAÇÃO}

O estudo realizado tem como questão de investigação: Que experiências pedagógicas com recurso a tecnologias têm sido realizadas em Portugal, entre 1975 e 2021, no Ensino Básico e Secundário?

Nesse sentido, foram delineadas algumas questões que serviram para a definição do corpus de artigos a selecionar:

Q1 - Que tecnologias foram sendo introduzidas nas escolas a partir de 1975?

Q2 - Que tipo de experiências pedagógicas foram sendo desenvolvidas pelos professores?

Q3 - Que opinião apresentam sobre o uso de tecnologias em contexto educativo? 
Q4 - Que relação existe entre a disseminação destas tecnologias e a formação docente para a sua utilização?

\subsection{ESCOLHA DAS FONTES DE ACESSO AOS DADOS}

Para recolha dos dados foram selecionadas as bases de dados da SCOPUS e da Web of Science, por se considerar que estas são espaços de divulgação científica e académica de excelência. Uma vez que após a primeira recolha de dados, estes nos remeteram apenas para textos mais recentes, foi adicionada outra base de dados, a da SCIELO, por considerarmos que poderia acrescentar dados relevantes, sobretudo no período referente às décadas iniciais do estudo.

\subsection{DEFINIÇÃO DAS PALAVRAS-CHAVE}

As palavras-chave definidas (em português e em inglês), procuraram englobar os diferentes aspetos a que se pretendia dar resposta, nomeadamente sobre a introdução das tecnologias e de diferentes recursos digitais, quer na perspetiva da educação em geral, quer relacionadas com o processo de ensino e aprendizagem, e também sobre competências digitais docentes, representando a Tabela 1 o número de resultados encontrados.

Tabela 1

Palavras-chave para pesquisa

\begin{tabular}{|c|c|c|c|c|}
\hline String & scopus & $\begin{array}{l}\text { Web of } \\
\text { Science }\end{array}$ & SCIELO & Total \\
\hline Technology AND Education & 520 & 99 & 204 & 823 \\
\hline Tecnologia AND Educação & 3 & 1 & 55 & 59 \\
\hline Technology AND Teaching & 244 & 21 & 149 & 414 \\
\hline Tecnologia AND Ensino & 1 & 0 & 70 & 71 \\
\hline Technology AND Learning & 270 & 74 & 175 & 519 \\
\hline Tecnologia AND Aprendizagem & 1 & 0 & 53 & 54 \\
\hline Digital AND Education & 158 & 54 & 181 & 393 \\
\hline Digital AND Educação & 0 & 0 & 66 & 66 \\
\hline Digital AND Teaching & 111 & 13 & 113 & 237 \\
\hline Digital AND Teaching & 1 & 1 & 71 & 73 \\
\hline Digital AND Learning & 147 & 62 & 149 & 358 \\
\hline \begin{tabular}{l|l} 
Digital AND Aprendizagem \\
\end{tabular} & 3 & 0 & 58 & 61 \\
\hline Audiovisual AND Education & 54 & 3 & 11 & 68 \\
\hline Audiovisual AND Educação & 1 & 0 & 2 & 3 \\
\hline Audiovisual AND Teaching & 21 & 0 & 11 & 32 \\
\hline Audiovisual AND Teaching & 0 & 1 & 4 & 5 \\
\hline Audiovisual AND Learning & 5 & 2 & 9 & 16 \\
\hline
\end{tabular}




\begin{tabular}{|c|c|c|c|c|}
\hline Audiovisual AND Aprendizagem & 0 & 0 & 4 & 4 \\
\hline Digital environment AND Education & 14 & 1 & 31 & 46 \\
\hline Ambiente Digital AND Educação & 0 & 0 & 4 & 4 \\
\hline Digital environment AND Teaching & 12 & 0 & 14 & 26 \\
\hline Ambiente Digital AND Teaching & 0 & 0 & 4 & 4 \\
\hline Digital environment AND Learning & 18 & 1 & 20 & 39 \\
\hline Ambiente Digital AND Aprendizagem & 0 & 0 & 4 & 4 \\
\hline Game AND Education & 113 & 32 & 26 & 171 \\
\hline Jogo AND Educação & 1 & 0 & 19 & 20 \\
\hline Game AND Teaching & 84 & 7 & 14 & 105 \\
\hline Jogo AND Teaching & 5 & 0 & 17 & 22 \\
\hline Game AND Learning & 113 & 66 & 23 & 202 \\
\hline Jogo AND Aprendizagem & 3 & 0 & 17 & 20 \\
\hline Digital competence & 7 & 6 & 27 & 40 \\
\hline Competência digital & 0 & 2 & 28 & 30 \\
\hline Digital literacy & 55 & 13 & 32 & 100 \\
\hline Literacia digital & 0 & 0 & 3 & 3 \\
\hline TOTAL & 1965 & 459 & 1668 & 4092 \\
\hline
\end{tabular}

\subsection{SELEÇÃO DOS ESTUDOS}

Os trabalhos foram selecionados de acordo com um conjunto de critérios de inclusão e de exclusão previamente definidos, tendo estes servido de base à análise dos títulos, resumos e palavras-chave dos artigos recolhidos. Um critério inicial de busca remeteu para a recolha exclusiva de artigos de revistas com peer review e relativos a atividades desenvolvidas em Portugal ou por autores portugueses no período temporal entre 1975 e 2021.

Critérios de inclusão:

Inc1- O trabalho relata experiências de utilização das tecnologias educativas em contexto educativo
Inc2- O trabalho apresenta uma reflexão teórica sobre a utilização das tecnologias educativas

Inc3- O trabalho discute as competências digitais necessárias para o uso de tecnologias em contextos educativos

Critérios de exclusão:

Exc1- O trabalho não diz respeito a estudos realizados em Portugal

Exc2-Trabalhos repetidos

Exc3- O trabalho não diz respeito à educação não superior

Exc4-Trabalhos sem acesso disponível 


\section{Tabela 2}

Resultados selecionados e excluídos ( $1^{a}$ etapa)

\begin{tabular}{|c|c|c|c|c|c|c|c|}
\hline \multirow{2}{*}{$\begin{array}{c}\text { Base de } \\
\text { dados }\end{array}$} & \multicolumn{7}{|c|}{ Excluídos } \\
\hline & & Exc1 & Exc2 & Exc3 & Exc4 & Exc5 & \\
\hline SCOPUS & 1965 & 1680 & 148 & 35 & 1 & 91 & 10 \\
\hline $\begin{array}{l}\text { Web of } \\
\text { Science }\end{array}$ & 459 & 10 & 155 & 71 & 5 & 172 & 46 \\
\hline SCIELO & 1668 & 1586 & 58 & 3 & 1 & 10 & 10 \\
\hline TOTAL & 4092 & 3276 & 361 & 109 & 7 & 273 & 66 \\
\hline
\end{tabular}

Exc5- Estudos com conteúdo irrelevante para o contexto da pesquisa deste trabalho

Depois de aplicados estes critérios foram selecionados 66 trabalhos entre o conjunto dos 4092 resultados encontrados, conforme se pode verificar na Tabela 2.

Numa segunda fase foi feita uma análise mais fina, tendo sido analisados os artigos na sua totalidade. Daí resultou a exclusão de dez textos da Web of Science, um da SCOPUS e um da
SCIELO, por se ter determinado que não se encontravam no contexto da pesquisa (Exc5); a exclusão de quatro textos da lista da Web of science por remeterem para conteúdos repetidos relativos aos mesmos projetos (Exc2); a exclusão de um texto da lista da Web of Science por não dizer respeito ao ensino não superior (Exc3); e excluídos dois textos da Web of Science, por não conterem informações relativas a Portugal (Exc1) (Tabela 3), Assim, excluídos os textos atrás referenciados o corpus a ser analisado traduziu-se num total de 47 artigos.

Tabela 3

Tabela final com os resultados selecionados e excluídos

\begin{tabular}{|c|c|c|c|c|c|c|c|}
\hline \multirow{2}{*}{$\begin{array}{l}\text { Base de } \\
\text { dados }\end{array}$} & \multirow{2}{*}{$\begin{array}{l}\text { Total de Pu- } \\
\text { blicações }\end{array}$} & \multicolumn{5}{|c|}{ Excluídos } & \multirow{2}{*}{$\begin{array}{l}\text { Total de trabalhos } \\
\text { selecionados }\end{array}$} \\
\hline & & Exc1 & Exc2 & Exc3 & Exc4 & Exc5 & \\
\hline SCOPUS & 1965 & 1680 & 148 & 35 & 1 & 92 & 9 \\
\hline $\begin{array}{c}\text { Web of Scien- } \\
\text { ce }\end{array}$ & 459 & 12 & 159 & 72 & 5 & 182 & 29 \\
\hline SCIELO & 1668 & 1586 & 58 & 3 & 1 & 11 & 9 \\
\hline TOTAL & 4092 & 3272 & 365 & 110 & 7 & 285 & 47 \\
\hline
\end{tabular}




\section{RESULTADOS}

Os textos selecionados foram analisados de forma rigorosa, com o objetivo de compreender quais os que se focavam mais no professor e quais os que se focavam mais no aluno. Concluiu-se que esta distribuição é quase equivalente (Tabela 6), com 24 textos com o foco no professor $(51,1 \%)$, 18 textos com foco no aluno $(38,3 \%)$ e 5 trabalhos $(10,6 \%)$ que articulam a relação professor-aluno nas experiências apresentadas.

Para além disso, verificou-se que 35 textos apresentam estudos empíricos, o que corresponde a $74,5 \%$ do total, enquanto apenas 12 textos $(25,5 \%)$ seguem uma metodologia de revisão bibliográfica ou de ensaio teórico.

No que concerne à ligação destes trabalhos com as questões de investigação, os trabalhos com foco nas aprendizagens dos estudantes refletem sobretudo experiências realizadas e os seus efeitos nessas mesmas aprendizagens e na modificação das práticas, cada vez mais associadas a produção de conhecimento, ou seja, articulando-se cada vez mais com uma ideia de "prod'utilização" de recursos ao serviço das aprendizagens.

No caso dos professores destaca-se o facto de existirem vários textos relativos à sua formação para o uso da tecnologia (34\%) e depois divide-se entre textos que apresentam diferentes tecnologias ou apresentam práticas realizadas com diferentes tecnologias digitais (Tabela 4).

Ressalva-se o facto de que existem dois textos incluídos na Q2 que já remetem para experiências durante o período da pandemia.
Tabela 4

Foco dos textos selecionados por questão de investigação

\begin{tabular}{|c|c|c|c|c|c|c|}
\multicolumn{3}{c}{ Aluno } & \multicolumn{5}{c|}{$\begin{array}{c}\text { Aluno e } \\
\text { Professor }\end{array}$} \\
\cline { 3 - 8 }$(N=47)$ & $F$ & $\%$ & $F$ & $\%$ & $F$ & $\%$ \\
\hline 01 & 10 & 21.3 & 7 & 14.9 & 3 & 6.4 \\
\hline 02 & 14 & 29.8 & 5 & 10.6 & 3 & 6.4 \\
\hline 03 & 16 & 34.0 & 8 & 17.0 & 4 & 8.5 \\
\hline 04 & 0 & 0.0 & 16 & $34.0 \%$ & 0 & 0.0 \\
\hline
\end{tabular}

\section{DISCUSSÃO}

\subsection{INTRODUÇÃO DE TECNOLOGIAS NAS ESCOLAS A PARTIR DO DESENVOLVIMENTO DOS COMPUTADORES}

Em Portugal, é sobretudo a partir dos anos 80 do século XX que se verifica um maior alcance das novidades tecnológicas associadas aos computadores e ao ensino programado, em parte resultado do desenvolvimento de programas governamentais, tal como os referidos no ponto 2 .

São vários os estudos dos anos 80 e 90 do século XX que mostram a introdução de um conjunto diversificado de equipamentos:

- ligados ao audiovisual, com novidades tecnológicas áudio e vídeo como os gravadores e leitores de cassetes e mais tarde CDs e DVDs;

- ligados à introdução dos computadores propriamente ditos nas escolas, em articulação com programas governamentais de apoio à aquisição destes equipamentos quer para as escolas, quer para professores e alunos; 
- ligados ao multimédia e, como refere Silva (1993), debruçando-se sobre a construção do cenário multidimensional e a representação cognitiva do conhecimento.

A internet e a rapidez das inovações tecnológicas do século XXI rapidamente encontraram o seu espaço nas salas de aula portuguesas. Primeiro, computadores, câmaras de vídeo, máquinas fotográficas, projetores e impressoras tornaram-se dispositivos existentes em muitas escolas, numa utilização sobretudo instrumental, "como objetivo de estudo específico em determinadas disciplinas [...], como ajuda ou ferramenta para o estudo de outras disciplinas [...] como apoio à atividade de gestão e administração, não especificamente docente" (Henriques et al., 2012, p. 11).

Mais recentemente foram sendo introduzidos os quadros interativos, os computadores portáteis (destacando-se aqui o exemplo do computador Magalhães), e as tecnologias móveis, como smartphones e tablets e mesmo a utilização de robots.

É necessário, porém, compreender de que forma estes equipamentos foram integrados nas práticas docentes.

\subsection{EXPERIÊNCIAS PEDAGÓGICAS DESENVOLVIDAS PELOS PROFESSORES}

Com o desenvolvimento das tecnologias, também os usos que delas vão sendo feitos se vão complexificando, com uma tónica no que os alunos podem fazer com estes diferentes recursos. Entre os textos selecionados, e como referido no ponto 3, os estudos apresentados têm sobretudo, esse foco no desenvolvimento das aprendizagens, no desenvolvimento de competências transversais e, também, reve- lando alguns deles preocupação com o desenvolvimento das competências digitais dos estudantes (Martins \& Fernandes, 2015; Pinto \& Osório, 2019) e da sua literacia mediática (Costa et al., 2018).

Apesar de alguns dos estudos serem realizados com níveis de escolaridade específicos, as competências trabalhadas e os objetivos previstos podem facilmente ser adaptados a outros ciclos de estudos, uma vez que se focam na utilização da tecnologia enquanto meio para o desenvolvimento de atividades quer disciplinares, quer transversais.

Os recursos apresentados nestes estudos são, de facto, bastante variados, desde, por exemplo, a utilização de livros digitais para desenvolvimento de competências ao nível da leitura e interpretação (Gonçalves \& Almeida, 2016), manipulativos digitais (Sylla et al. 2015), podcasts (Coutinho \& Mota, 2011) ou redes sociais que potenciam o desenvolvimento de aprendizagens informais (Moreira et al., 2019).

Certo é que a gamificação é a estratégia mais apresentada (representando $41 \%$ do foco dos textos que apresentam experiências pedagógicas), com ou sem recurso a tecnologias móveis e, mais recentemente, incluindo mesmo uma nova forma de interação: a realidade aumentada que, como referido por Estudante e Dietrich (2020), se tem tornado cada vez mais uma realidade, fruto sobretudo da democratização das tecnologias móveis.

De facto, tablets e smartphones encontraram mesmo o seu caminho no auxílio ao desenvolvimento de atividades de ensino e de aprendizagem, surgindo claramente referidos em 27\% das experiências relatadas nestes estudos.

Há exemplos entre os estudos selecionados, que demonstram bem a diversidade de possibilidades que a complexificação quer dos equi- 
pamentos, quer do próprio software têm vindo a permitir, por exemplo com a realidade aumentada e os manipulativos digitais (Sylla et al., 2015) ou o recurso a atividades de storytelling como as apresentadas por Rodrigues e Bidarra (2014) que recorrem a diferentes plataformas para a concretização da atividade.

Contudo, como Almeida (2018) refere, há ainda vários professores que utilizam as tecnologias digitais como suporte ao ensino e não tanto como apoio às aprendizagens, em práticas que tivessem vindo alterar significativamente a abordagem pedagógica, nem trazer uma verdadeira inovação ou reconfiguração do processo educativo.

\subsection{OPINIÃO SOBRE O USO DE TECNOLOGIAS EM CONTEXTO EDUCATIVO}

Entre os textos analisados verifica-se uma tendência (28,6\% dos textos) para relacionar as tecnologias quer com níveis de motivação e satisfação elevados (Barros et al., 2020; Gonçalves \& Almeida, 2016; Faria et al., 2019), quer com aprendizagens mais eficazes (22,5\% dos textos) (Santos \& Alves, 2017).

Os aspetos positivos são, de facto, variados, considerando-se a importância de que estas participações ativas dos estudantes ajudam a aumentar um sentimento de autovalorização (Pinto \& Osório, 2020), a uma melhor comunicação entre os atores do processo educativo (Fartura et al., 2014) e também na partilha de conhecimento (Moreira et al., 2019). Como Gonçalves e Almeida (2016) indicam, configuram-se novas formas de ensinar, mas também novas formas de aprender.

Costa et al. (2020) destacam que, por exemplo, a utilização de realidade aumentada contribui para uma experiência diferente, aproximando mesmo os alunos de uma "experiência real", aliando-se o lado lúdico destas estratégias à aprendizagem.

É de destacar também as referências de Fernandes et al. (2016) e de Moniz et al. (2021), no que diz respeito à importância do docente enquanto mediador de todas estas atividades, não só na sua planificação, mas também na sua implementação.

\subsection{RELAÇÃO ENTRE A DISSEMINAÇÃO DESTAS TECNOLOGIAS E A FORMAÇÃO DOCENTE}

A falta de formação docente tem sido muito apontada como uma justificação para uma utilização incipiente, conservadora ou instrumental das tecnologias na sala de aula sendo, por isso, reconhecida como essencial.

O facto de o Estatuto da Carreira Docente garantir ao docente o acesso regular a formação contínua para atualização e aperfeiçoamento dos seus conhecimentos e das suas competências profissionais e, sobretudo desde 2007, o facto da formação contínua em TIC ter sido considerada prioritária (Ricoy \& Couto, 2011), aumentou bastante a oferta e, naturalmente, a participação.

Porém, a formação inicial de professores não obriga à existência de unidades curriculares relacionadas com as tecnologias educativas, ainda que a legislação indique que a formação inicial deva incluir uma área de formação educacional geral que abranja "os conhecimentos, as capacidades e as atitudes comuns a todos os docentes relevantes para o seu desempenho na sala de atividades ou na sala de aula," (Decreto-Lei n. ${ }^{\circ}$ 79/2014, de 14 de maio, art. ${ }^{\circ}$ $9^{\circ}$, p. 2891). Deste modo, apesar de a preocupação com a formação inicial docente ser uma realidade (Aşık, et al., 2020; Gutiérrez-Fallar \& Henriques, 2020; Ricoy \& Couto, 2011; Rodri- 
gues, 2020), constata-se, porém, que ainda é insuficiente (Ricoy \& Couto, 2011).

No que diz respeito então à formação para uma utilização das tecnologias, Gutiérrez-Fallas e Henriques (2020) e Sampaio (2016) destacam a necessidade de organizar a formação de acordo com o modelo TPACK, "apresentando preocupações quer pedagógicas quer tecnológicas, considerando o contexto de cada professor e respetivos alunos" (Sampaio, 2016, p. 223)

Para além disso, a preferência por formações que tenham uma duração relativamente alargada no tempo também é frequente, porque permite compreender melhor o efeito que tem na prática docente. Assim, os autores destes trabalhos conseguem verificar que os formandos começam a modificar as suas práticas, promovendo aulas mais ativas e dinâmicas e que começam a fazer uso das tecnologias como meio para o desenvolvimento de estratégias inovadoras, dinâmicas e promotoras de aprendizagens ativas (Carlos et al., 2018; Montez \& Aires, 2013; Sampaio, 2016), apesar de considerarem que estas mudanças não se concretizam de um momento para o outro, mas sim que se tratam de lentos processos que precisam "de uma certa maturação para gerar transformação" (Sampaio, 2016, p. 222).

Mais recentemente, tem sido mais evidente a relevância do tema das competências digitais docentes, sendo o referencial DigCompEdu, elaborado pela União Europeia, um dos mais destacados. A partir deste referencial, a União Europeia preparou um questionário de autoavaliação das competências digitais docentes (o DigCompEdu CheckIn), que foi posteriormente validado para a população portuguesa por Dias-Trindade et al. (2019). Da mesma forma que Sampaio (2016) refere a importância de que a formação seja mais focada em áreas científicas específicas, ou numa articulação entre a pedagogia, o conteúdo e a tecnologia, o que este questionário vem permitir, como referido por Dias-Trindade e Ferreira (2020), é que cada professor possa "definir qual das formações deve realizar, investir até primeiro naquelas onde encontrou maiores dificuldades e ir avançando à medida dos seus interesses, evoluindo, desta forma, ao seu ritmo, a caminho da obtenção da fluência digital" (p. 181).

O estudo realizado em Portugal a partir deste questionário (Dias-Trindade \& Moreira, 2020) mostrou, por exemplo, maiores fragilidades ao nível de competências mais articuladas com a adaptação às diferentes necessidades dos alunos. Quando se referem ao feedback, à aprendizagem autorregulada, à adaptação da aprendizagem, ou seja, ao trabalho prático de acordo com as necessidades dos alunos, surgem maiores dificuldades na adaptação ao contexto digital. Por outro lado, as competências relacionadas com o trabalho individual de um professor, com uma prática reflexiva e de planeamento, são as que coletam, em média, os resultados mais elevados (Dias-Trindade \& Moreira, 2020). Já o estudo de Ricoy e Couto (2011), também a partir do referencial DigCompEdu, mostra que, entre participantes ainda em formação inicial de professores, existem algumas lacunas nas práticas que se relacionam com a segurança digital.

Feita esta reflexão, importa ainda destacar os dois textos publicados já em contexto de pandemia. Dias-Trindade et al. (2020) traçam um panorama sobre a transição de um regime presencial para a educação remota emergencial em Portugal (comparando também com o Brasil). Por sua vez Moreira et al. (2020) reforçam a necessidade de formação e capacitação docente para os contextos educativos digitais. 


\section{CONCLUSÕES}

Com os avanços tecnológicos dos últimos cerca de 60 anos os computadores foram-se tornando cada vez mais potentes e portáteis, com o aparecimento da Internet passaram a poder estar conectados e, mais recentemente, a eles se foram associando novos equipamentos, digitais, móveis, que permitem, conforme reconhecem os estudos analisados, uma maior interatividade e participação a partir das metodologias que os docentes podem promover.

Porém, muitos dos autores reconhecem que a utilização que se tem feito das tecnologias que têm entrado nas escolas (computadores, projetores, quadros interativos, tablets, entre outros) tem sido conservadora e pouco tem sido associada a práticas inovadoras. Mas, indicam também que para que a renovação aconteça é necessário apostar em formação para capacitar e dotar os professores de competências digitais que Ihes permitam utilizar as tecnologias com intencionalidade pedagógica.

Nesse sentido, muitos têm sido os projetos que, desde os anos 80 , os governos portugueses têm desenvolvido, em associação com projetos europeus, para capacitar digitalmente os professores e mesmo para equipar as escolas e a comunidade escolar. Porém, pela análise da amostra recolhida para este estudo, verifica-se que ainda há um longo caminho a percorrer que, para vários autores, deve começar pela formação inicial de professores.

Em termos gerais o que estes trabalhos deixam transparecer é a evolução que o papel da tecnologia tem tido em contexto educativo, ainda que assumidamente mais lenta do que seria desejável. No entanto, sobretudo, nos últimos 20 anos tem vindo a assistir-se a uma utilização mais efetiva da tecnologia por parte dos alunos, visando aprendizagens cada vez mais ativas e participadas, que nos pode levar a considerar que esta etapa mais recente da relação das tecnologias com a escola está a converter-se numa era dos "prod'utilizadores", onde os alunos interagem com as tecnologias, em conjunto com os seus professores, para aprender e alargar os seus conhecimentos contribuindo, também, com a produção de novos conteúdos numa constante interação entre produção e utilização. Como Oliveira (2020) refere, é fundamental pensar numa "Escola que faz aprender, em vez de ensinar, onde o aluno é construtor do seu projeto de vida. [...] Uma Escola onde as competências digitais são transversais e onde o aluno é utilizador/produtor de tecnologia" ( $p$. 497). Daí a importância, também, do desenvolvimento das competências digitais não só dos professores, mas também dos estudantes.

A pandemia iniciada em 2020 veio colocar mais visíveis as necessidades e acelerar o processo de digitalização das escolas, processo esse que estava já em curso em Portugal enquadrado no Portugal INCoDe.2030 (Iniciativa Nacional Competências Digitais e.2030), iniciado em março de 2017, e que em abril de 2020, na senda de capacitação digital do país, vem reforçar a aposta na Educação Digital através da Resolução do Conselho de Ministros n. ${ }^{0}$ 30/2020. Esta resolução aprova o Plano de Ação para a Transição Digital, que inclui uma área estratégica focada na "Capacitação e inclusão digital das pessoas", através da Educação Digital, e prevê um programa de digitalização para as escolas que inclui, para além do acesso a recursos e equipamentos digitais, a aposta num plano de capacitação digital dos docentes.

Em linha com a ideia de uma escola digital, onde os processos de ensino e de aprendizagem sejam cada vez mais enriquecidos pelas tecnologias digitais, e onde humanos e não humanos interagem, colaborativamente, com o intuito de ensinar, de aprender, de construir conheci- 
mento, de forma ativa e participada é o objetivo para a escola portuguesa, uma escola que faça uso da tecnologia e do digital para aproximar "Os alunos das ferramentas de produtividade e colaboração que podem encontrar num ambiente de trabalho profissional" (Resolução do Conselho de Ministros n. ${ }^{\circ}$ 30/2020, p. 15), ou seja, uma escola que se desenvolve em pleno numa era de "prod'utilizadores".

\section{REFERENCIAS}

Almeida, P. (2018). Tecnologias digitais em sala de aula: o professor e a reconfiguração do processo educativo. Da Investigação às Práticas, 8(1), $4-21$.

Alves, L., Torres, V., Neves, I., \& Fraga, G. (2019). Tecnologias digitais nos espaços escolares: um diálogo emergente. In O. Ferraz (org.). Educação, (multi) letramentos e tecnologias: tecendo redes de conhecimento sobre letramentos, cultura digital, ensino e aprendizagem na cibercultura (pp. 117140). EDUFBA.

Aşık, A., Köse, S., Gonca Yangın, E., Seferoğlu, G., Pereira, R., \& Ekiert, E. (2020). ICT integration in English language teacher education: insights from Turkey, Portugal and Poland. Computer Assisted Language Learning, 33(7), 708-731. https://www.doi.org/10.1080/09588221.2019.158874 4

Barros, C., Carvalho, A. A., \& Salgueiro, A. (2020). The effect of the serious game Tempoly on learning arithmetic polynomial operations. Education and Information Technologies, 25, 1497-1509. https://www.doi. org/10.1007/s10639-019-09990-4

Bassani, P. B. S., \& Barbosa, D. N. F. (2018). Experiences with Web 2.0 in school settings: a framework to foster educational practices based on a personal learning environment perspective. Educação em Revista, 34, 34:e162010. http://dx.doi.org/10.1590/0102-4698162010

Carlos, V., Pombo, L., \& Loureiro, M. J. (2018). Integração pedagógica das TIC no contexto de um Edulab reflexão e sistematização de princípios orientadores de boas práticas (projeto AGIRE). Da Investigação às Práticas, 8(1), $22-41$.

Conselho de Ministros (2020). Resolução n 30/2020. Diário da República $n^{\circ}$ 78/2020, Série I de 2020-04-21. pp. 6 - 32. 
Costa, C., Tyner, K., Henriques, S., \& Sousa, C. (2018). Game Creation in Youth Media and Information Literacy Education. International Journal of Game-Based Learning, 8(2), mar, 1-13.

Costa, M. C., Manso, A., \& Patrício, J. (2020). Design of a Mobile Augmented Reality Platform with Game-Based Learning Purposes. Information, 11, 1-19. https://www.doi.org/10.3390/info11030127

Dias-Trindade, S., Correia, J., \& Henriques, S. (2020). O ensino remoto emergencial na educação básica brasileira e portuguesa: a perspectiva dos docentes. Revista Tempos e Espaços em Educação. 13(32), jan/dez. http:// dx.doi.org/10.20952/revtee.v13i32.14426

Dias-Trindade, S., \& Ferreira, A. G. (2020). Digital teaching skills: DigCompEdu Checkln as an evolution process from literacy to digital fluency. ICON014, 18(2), 162-187. https://www.doi.org/10.7195/ri14.v18i1.1519

Dias-Trindade, S., \& Moreira, J. A. (2020). Assessment of high school teachers on their digital competences. MAGIS, Revista Internacional de Investigación em Educación, 13, 1-21. https://www.doi.org/10.11144/Javeriana. m13.ahst

Dias-Trindade, S., Moreira, J. A., \& Nunes, C. (2019). Escala de autoavaliação de competências digitais de professores. Procedimentos de construção e validação. Texto Livre, 12(2), mai/ago, 152-171. https://www. doi.org/10.17851/1983-3652.12.2.152-171

Estudante, A., \& Dietrich, N. (2020). Using Augmented Reality to Stimulate Students and Diffuse Escape Game Activities to Larger Audiences. J. Chem. Educ., 97, 1368-1374. https://www.doi.org/10.1021/acs. jchemed.9b00933

Faria, E., Guilherme, E., Pintassilgo, J., Mogarro, M. J., Pinho, A. S., Baptista, M., Chagas, I., \& Galvão, C. (2019). The Portuguese Maritime Voyages of Discovery: the exploration of the history of a city with an App as an educational resource. Digital Education Review, 36, dez, 85-99.

Fartura, S., Pessoa, T., \& Barreira, C. (2014). El papel de las tic en las prácticas de los profesores de educación primaria en Portugal. Estudio exploratorio. Profesorado: revista de currículum y formación del professorado, 18(3), set-dez, 119-135.

Fernandes, L. M. A., Matos, G. C., Azevedo, D., Nunes, R. R., Paredes, H., Morgado, L., Barbosa, L. F., Martins, P., Fonseca, B., Cristóvão, P., Carvalho, F., \& Cardoso, B. (2016). Exploring educational immersive videogames: an empirical study with a 3D multimodal interaction prototype. Behaviour \& Information Technology, 35(11), 907-918. https://www.doi. org/10.1080/0144929X.2016.1232754

Figueiredo, A. D. (1989). Computadores nas escolas. Revista Colóquio-Ciências. Jan-abr, 76-89. 
Gonçalves, D., \& Almeida, S. (2016). Learning and teaching using digital books: opportunities and constraints. Profesorado: Revista de Currículum y Formación del professorado. 20(1), jan/abr, 49-60.

Governo Português (2017). Portugal INCoDe.2030: iniciativa nacional de competências digitais e.2030. República Portuguesa. https://www.incode2030. gov.pt/sites/default/files/incode2030_pt.pdf

Gutiérrez-Fallas, L. F., \& Henriques, A. (2020). O TPACK de futuros professores de matemática numa experiência de formação. Revista Latinoamericana de Investigación en Matemática Educativa, 23(2), 175-202. https:// www.doi.org/10.12802/relime.20.2322

Henriques, S., Moreira, A., Fombona, J., \& Barros, D. (2012). As TIC no contexto educativo português. Revista EDAPICI, 12(12), dezembro, 6-26.

Martins, S., \& Fernandes, E. (2015). Robots como ferramenta pedagógica nos primeiros anos a aprendizagem como participação. Revista Brasileira de Educação, 20(61), abr/jun, 333-358. https://www.doi.org/10.1590/ S1413-24782015206104

Ministério da Economia e da Transição Digital (2020). Portugal digital: moving forward. Moving with a purpose. Ministério da Economia e Transição Digital. https://www.portugal.gov.pt/gc22/portugal-digital/plano-deacao-para-a-transicao-digital-pdf.aspx

Moniz, A. B., Dias-Trindade, S., \& Neto, M. (2021). "Pedagogia da memória" no século digital: tecnologias digitais para estudar história local. EducaOnline, 15(1), 153-174.

Montez, R., \& Aires, M. L. L. (2013). Colaboración online, formación del profesorado y TIC en el aula: estudio de caso. Teoría de la Edicación: Educación y Cultura em la Sociedad de la Información, 14(3), 277-301.

Moreira, J. A., Henriques, S., \& Barros, D. (2020). Transitando de um ensino remoto emergencial para uma educação digital em rede, em tempos de pandemia. Dialogia, 34, 351-364. https://www.doi.org/10.5585/Dialogia.N34.17123

Moreira, J. A., Santana, C., \& Bengoechea, A. (2019). Ensinar e aprender nas redes sociais digitais: o caso da Mathgurl no Youtube. Revista de Comunicación de la SEECl, 50, nov/mar, 107-127.

Oliveira, A. (2020). Ensino profissional: 30 anos a coconstruir projetos de vida. In M. Miguéns (coord.). Estado da Educação 2019 (pp. 494-505). Conselho Nacional de Educação.

Petticrew, M., \& Roberts, H. (2006). Systematic reviews in the social sciences: a practical guide. Blackwell Publishing. 
Pinheiro, B., \& Correia, L. G. (2014). E-learning: introdução histórica a uma tecnologia sempre renovada em contexto educativo. In F. Vieira e M. T. Restivo (Org.) Novas tecnologias e educação: ensinar a aprender/aprender a ensinar (pp. 45-104). Biblioteca Digital da Faculdade de Letras da Universidade do Porto.

Pinto, M., \& Osório, A. (2019). Aprender a programar en educación infantil: análisis com la escala de participación. Píxel-BIT Revista de Medios y Educación, 55, 133-156. https://www.doi.org/10.12795/pixelbit.2019. i55.08

Rodrigues, A. L. (2020). Digital technologies integration in teacher education: the active teacher training model. Journal of e-learning and knowledge society, 16(3), 24-33. https://www.doi.org/10.20368/1971-8829/1135273

Rodrigues, P., \& Bidarra, J. (2014). Transmedia Storytelling and the Creation of a Converging Space of Educational Practices. IJET, 9(6), 42-48. https:// www.doi.org/10.3991/ijet.v9i6.4134

Rosenberg, M. J. (2001), E-learning strategies for delivering knowledge in digital age. McGraw-Hill.

Russell, M. (2006). Technology and assessment: the tale of two interpretations. Information Age Publishing.

Ricoy, M. C., \& Couto, M. J. V. S. (2011). As TIC no ensino secundário na matemática em Portugal: a perspectiva dos professores. Revista Latinoamericana de Investigación en Matemática Educativa, 14(1), 95-119.

Saettler, P. (2004). The Evolution of American Educational Technology. Information Age Publishing.

Sampaio, P. (2016). Desenvolvimento profissional dos professores de Matemática: Uma experiência de formação em TIC. Revista Portuguesa de Educação, 29(2), 209-232. https://www.doi.org/10.21814/rpe.2987

Santos, T., \& Alves, M. P. (2017). O contributo das tecnologias no desenvolvimento do currículo escolar: perspectivas dos alunos. RIAEE - Revista Ibero-Americana de Estudos em Educação, 12(2), 1554-1569. https:// www.doi.org/10.21723/riaee.v12.n.esp.2.10309

Sarramona, J. (1986). Prólogo. In J. Castillejo; A. Colom; J. Escamez; J. Garcia Carrasco; A. Sanvicens; J. Sarramona e G. Vázquez. Tecnología y Educación (pp. 9-10). Ediciones Ceac S.A.

Selwyn, N. (2010). Web 2.0 and the school of the future, today", in OCDE (org.) Inspired by Technology, Driven by Pedagogy: A Systemic Approach to Technology-Based School Innovations (pp. 23-43). OECD Publishing. https://doi.org/10.1787/9789264094437-4-en 
Silva, B. (1989). Os recursos didácticos na rede escolar do distrito de Braga. Revista Portuguesa de Educação, 2(2), 107-127.

Silva, B. (1993). Tecnologia educativa em Portugal: conceito, origens, evolução, áreas de intervenção e investigação. Revista Portuguesa de Educação, 6(3), 37-55.

Silva, B. (2001). As tecnologias de informação e comunicação nas reformas educativas em Portugal. Revista Portuguesa de Educação, 14(2), 111-153.

Skinner, B. F. (1958). Teaching Machines. Science (nova série). 128(3330), 969977.

Sylla, C., Pereira, I. S. P., Coutinho, C. P., \& Branco, P. (2015). Digital Manipulatives as Scaffolds for Preschoolers' Language Development. IEEE Transactions on Emerging Topics In Computing, 4(3), 439-449. https://www.doi. org/10.1109/tetc.2015.2500099

Thammishetty, M. (ed.) (2015). Educational Technology. Laxmi Book Publication.

Unwin, D., \& McAleese, R. (1978). The encyclopaedia of educational media communications and technology. The Macmillan Press Ltd. 